\title{
Requeijão cremoso tradicional e light: Aceitabilidade e correlação das medidas sensoriais e instrumentais
}

\author{
Traditional and light processed cheese: Acceptability and correlation of sensory and instrumental
} measures

Queso procesado tradicional y ligero: Aceptabilidad y correlación de medidas sensoriales e instrumentales

Felipe Sousa da Silva

ORCID: https://orcid.org/0000-0002-1166-8474 Instituto Federal de Educação, Ciência e Tecnologia do Ceará, Brasil E-mail: fesosi2005@gmail.com

Sheyla Maria Barreto Amaral ORCID: https://orcid.org/0000-0003-0041-5487 Instituto Federal de Educação, Ciência e Tecnologia do Ceará, Brasil E-mail: sheylaamaral82@gmail.com

Ana Paula Ferreira de Almeida ORCID: https://orcid.org/0000-0002-3573-2987 Instituto Federal de Educação, Ciência e Tecnologia do Ceará, Brasil E-mail: ana.paula.ferreira.almeida123@gmail.com

Renata Chastinet Braga

ORCID: https://orcid.org/0000-0001-6630-2835 Instituto Federal de Educação, Ciência e Tecnologia do Ceará, Brasil E-mail: rchastinet@ifce.edu.br

Marlene Nunes Damaceno ORCID: https://orcid.org/0000-0002-3553-6740 Instituto Federal de Educação, Ciência e Tecnologia do Ceará, Brasil E-mail: marlene@ifce.edu.br

\begin{abstract}
Resumo
A aceitabilidade do requeijão está relacionada com a textura que entre outros fatores, recebe influência do teor de gordura. O objetivo desse estudo foi analisar a aceitabilidade sensorial de duas marcas comerciais de requeijão cremoso tradicional e light (TA, TB, LA, LB) e realizar a correlação com medidas instrumentais da textura. A avaliação sensorial foi realizada com 121 avaliadores não treinados, utilizando os testes de aceitação por escala hedônica estruturada de 9 pontos para os atributos impressão global, aparência, odor, textura e sabor, e por Check-AllThat-Apply (CATA) com 12 termos descritores. Para avaliação do Perfil de Textura Instrumental (TPA) realizou-se três ensaios em triplicata em analisador de textura. As quatro amostras obtiveram aceitação com médias dos escores entre 6,45 e 7,74 e Índice de Aceitabilidade superior a 70\% para todos os atributos. Os avaliadores identificaram diferença significativa entre os requeijões para cada termo sensorial abordado no CATA. Para TPA, o único parâmetro que não apresentou diferença $(\mathrm{p} \geq 0,05)$ foi a elasticidade. A Análise de Correspondência explicou 98,82\% da variação. A correlação das medidas sensoriais e instrumentais da textura indicou que a marca $\mathrm{B}$, independente do processo, apresenta as mesmas características. Na marca A, foi perceptível a influência do teor de gordura no processamento, pois os avaliadores distinguiram o requeijão LA do TA, evidenciando que o processo de fabricação permitiu a diferenciação quanto à textura e os atributos sensoriais.
\end{abstract}

Palavras-chave: CATA; Consumo; Derivados lácteos; Escala hedônica; TPA.

\begin{abstract}
The acceptability of the processed cheese is related to the texture which, among other factors, is influenced by the fat content. The aim of this study was to analyze the sensory acceptability of two commercial brands of traditional and light processed cheese (TA, TB, LA, LB) and perform the correlation with instrumental measurements of texture. The sensory evaluation was performed with 121 untrained panelists, using the acceptance tests by hedonic scale of 9 points for attributes overall impression, appearance, smell, texture and flavor and Check-All-That-Apply (CATA) with 12 descriptive terms. To evaluate the Instrumental Texture Profile (TPA), three tests were performed in triplicate on a texture analyzer. The four samples obtained acceptance with mean scores between 6.45 and 7.74 and an Acceptability Index greater than $70 \%$ for all attributes. The sensory assessors identified a significant difference
\end{abstract}


between the processed cheese for each sensory term covered in the CATA. For TPA, the only parameter that showed no difference $(\mathrm{p} \geq 0.05)$ was elasticity. Correspondence Analysis explained $98.82 \%$ of the variation. The correlation of sensory and instrumental measurements indicated that the texture of brand $\mathrm{B}$, regardless of the process has the same characteristics. In the A brand, the influence of the fat content in the processing was noticeable, as the evaluators distinguished the processed cheese LA from the TA, showing that the manufacturing process allowed the differentiation in terms of texture and sensory attributes.

Keywords: CATA; Consumption; Dairy derivatives; Hedonic scale; TPA.

\section{Resumen}

La aceptabilidad del queso procesado está relacionada con la textura que, entre otros factores, es influenciada por el contenido de grasa. El objetivo de este estudio fue analizar la aceptabilidad sensorial de dos marcas comerciales de queso procesado tradicional y ligero (TA, TB, LA, LB) y realizar la correlación con medidas instrumentales de textura. La evaluación sensorial se realizó con 121 jueces no capacitados, utilizando las pruebas de aceptación por escala hedónica de 9 puntos para los atributos impresión global, apariencia, olor, textura y sabor y por Check-AllThat-Apply (CATA) con 12 términos descriptivos. Para evaluar el Perfil de Textura Instrumental (TPA), se realizaron tres pruebas por triplicado en un analizador de textura. Las cuatro muestras obtuvieron aceptación con puntuaciones medias entre 6,45 y 7,74 y un índice de aceptabilidad superior al 70\% para todos los atributos. Los catadores identificaron una diferencia significativa entre el queso procesado para cada término sensorial cubierto en la CATA. Para el TPA, el único parámetro que no mostró diferencia $(\mathrm{p} \geq 0,05)$ fue la elasticidad. El Análisis de Correspondencia explicó el 98,82\% de la variación. La correlación de medidas sensoriales e instrumentales indicó que la textura de la marca B, independientemente del proceso, tiene las mismas características. En la marca A se notó la influencia del contenido de grasa en el procesamiento, ya que los evaluadores distinguieron el queso procesado LA del TA, mostrando que el proceso de elaboración permitió la diferenciación en cuanto a textura y atributos sensoriales.

Palabras clave: CATA; Consumo; Derivados lácteos; Escala hedónica; TPA.

\section{Introdução}

O Brasil é responsável por uma produção ampla de diferentes tipos de queijos, representando os hábitos e costumes da população brasileira. Um estudo realizado entre os meses de abril e maio de 2020, com mais de 5 mil consumidores, avaliou o comportamento em relação ao consumo de leites e derivados, observando que os queijos são os derivados lácteos mais adquiridos, representando cerca de 97\% (Embrapa, 2020a).

No Brasil, o requeijão é o tipo de queijo com crescente aumento de produtividade, dados de uma pesquisa de mercado realizado em 2013 mostram que foram fabricadas 182 mil toneladas de requeijão culinário correspondendo a 20,4\%, e 77,868 mil toneladas de requeijão cremoso equivalente a 8,7\% (Trintim et al., 2017). Já no ano de 2019, a produção de requeijão apresentou um aumento de aproximadamente $20 \%$, no que diz respeito ao índice de alcance, tornando-o mais popular no país (Embrapa, 2020b).

Em uma visão geral, o requeijão cremoso é um derivado lácteo de fácil elaboração e grande aceitação pelos consumidores, tendo relação com sua utilidade em diversos meios, entre eles o uso na culinária. Esse produto permite algumas variações durante seu processo e mudanças tecnológicas (Masson, Viganó \& Bordignon, 2016).

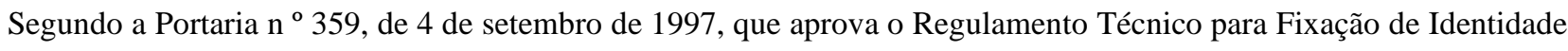
e Qualidade do Requeijão, este é definido como produto obtido pela fusão da massa coalhada, cozida ou não, dessorada e lavada, obtida por coagulação ácida e/ou enzimática do leite opcionalmente adicionada de creme de leite e/ou manteiga e/ou gordura anidra de leite ou butter oil. O requeijão pode ser classificado de acordo com a matéria prima e ingredientes usados, como por exemplo o requeijão cremoso tradicional, que apresenta na sua composição leite ou leite reconstituído, creme e ou manteiga e ou gordura anidra de leite ou butter oil, e o requeijão light com quantidade reduzida do teor de gordura (Brasil, 1997).

A aceitabilidade do requeijão está relacionada com sua textura que recebe influência do teor de gordura. Amaral et al. (2020), em estudo sobre avaliação das características físico-químicas e microbiológicas de quatro marcas de requeijão cremoso tradicional, não encontraram diferença em relação ao teor de gordura. Enquanto, Lima et al. (2021) no estudo sobre o perfil 
físico-químico e sensorial de requeijão cremoso obtido a partir de diferentes coagulantes (ácido acético, cítrico e lático), utilizando o Check-All-That-Apply (CATA) como um dos testes sensoriais, observaram uma maior aceitação entre os requeijões cremosos, principalmente para o atributo textura.

Contudo, é importante realizar a avaliação sensorial de produtos para caracterização de seus atributos sensoriais, como por exemplo a textura, sendo fundamental para transmitir informações destes e atender as preferências do consumidor (Alcantara \& Freitas-Sá, 2018), mas ao mesmo tempo, é necessário que se determine a textura por meio instrumental utilizando texturômetros, que avaliam as características reológicas dos alimentos apresentando similaridade em relação a prática de degustação, pois esses instrumentos são considerados precisos, rápidos e simples, auxiliando a correlação de resultados do teste instrumental com a avaliação sensorial (Souto, Santos, Alves, Nascimento \& Silva, 2017).

Portanto, essa correlação é de suma importância para a indústria de alimentos, pois a textura é considerada pelos consumidores um atributo sensorial fundamental para a qualidade de um produto disponível no mercado. Diante disso, o objetivo do presente estudo foi analisar a aceitabilidade sensorial de duas marcas comerciais de requeijão cremoso tradicional e light e realizar a correlação entre as medidas sensoriais e instrumentais no que diz respeito a textura.

\section{Metodologia}

A pesquisa se classifica com finalidade tecnológica, de natureza experimental e abordagem quali-quantitativa, apresentando objetivos descritivos, procedimentos técnicos de laboratório e desenvolvimento transversal (Cervo, Bervian \& Da Silva, 2007; Pereira, Shitsuka, Parreira \& Shitsuka, 2018;). Os dados foram organizados estatisticamente, possibilitando uma melhor compreensão dos resultados obtidos.

\subsection{Amostras de Requeijão}

As amostras comerciais de requeijão cremoso fabricadas pelos processos tradicional e light (TA, TB, LA, LB) foram adquiridas em supermercados da cidade de Limoeiro do Norte, Estado do Ceará. Todas as amostras pertenciam ao mesmo lote, e foram acondicionadas em embalagens de isopor, encaminhadas até o laboratório, sendo mantidas sob refrigeração $\left(5^{\circ} \mathrm{C} \pm 1\right.$ $\left.{ }^{\circ} \mathrm{C}\right)$ até o momento da realização das análises.

\subsection{Avaliação Sensorial}

A pesquisa foi aprovada pelo Comitê de Ética em Pesquisa do Instituto Federal de Educação, Ciência e Tecnologia do Ceará - IFCE sob Parecer n 3.691.340, respeitando a Resolução n466/2012 do Ministério da Saúde, referente a pesquisas envolvendo seres humanos (Brasil, 2013).

Os testes sensoriais foram realizados no laboratório de análise sensorial do IFCE Campus Limoeiro do Norte, seguindo as metodologias propostas por Dutcosky (2013). A cada avaliador foram servidos $20 \mathrm{~g}$ das quatro amostras à temperatura aproximada de $22{ }^{\circ} \mathrm{C}$, em recipientes descartáveis e codificados, em ordem de apresentação balanceada, acompanhados de água e biscoito, para limpeza do paladar, e a ficha-teste.

Os analisadores avaliaram o produto por teste de escala hedônica estruturada cujas notas variavam de 1 a 9, sendo: 1 desgostei muitíssimo, 5 - nem gostei, nem desgostei, e 9 - gostei muitíssimo, para os atributos impressão global, aparência, odor, textura e sabor; e por análise descritiva quantitativa pelo teste CATA (Check-All-That-Apply), utilizando 12 termos descritores para o produto, sendo 3 termos associados com os atributos hedônicos - Aparência: Cor branca, Cor amarela, Massa homogênea; - ㅇor: Ácido/azedo, Característico de requeijão, Aroma de queijo; Textura: Gorduroso, Cremoso, Firme; Sabor: Salgado, Ácido, Adocicado. 


\subsection{Perfil Instrumental de Textura}

Para avaliação do Perfil de Textura Instrumental (TPA) as amostras foram submetidas a três ensaios em triplicata em um analisador de textura TA.XT EXPRESS (Stable Micro Systems) equipado com uma célula de $10 \mathrm{~kg}$. $\mathrm{O}$ teste foi conduzido a temperatura de aproximadamente $25^{\circ} \mathrm{C}$. Para comprimir as amostras contidas em cápsulas de alumínio, utilizou-se um cilindro de aço inoxidável (probe) de $36 \mathrm{~mm}$ de diâmetro (SMS P/36 R). As condições do teste foram: célula de carga de $1 \mathrm{KN}$, distância de compressão de $20 \%$ da altura do produto, velocidade: $1,0 \mathrm{~mm} / \mathrm{s}$, tempo de contato de 10 segundos, com 2 ciclos de penetração e 6 repetições.

A programação dos testes e coleta de dados foram realizados pelo programa computacional Exponent Lite Express (Stable Micro Systems). A partir da curva obtida (força $\mathrm{x}$ tempo) foram calculados os resultados para firmeza (N), adesividade $(\mathrm{J})$, elasticidade $(\mathrm{mm})$, coesividade (adimensional), gomosidade $(\mathrm{N})$ e mastigabilidade $(\mathrm{J})$ das amostras, conforme Bourne (1978). Este perfil de textura instrumental foi comparado com o perfil de textura sensorial, para definir valores médios do instrumento para a textura ideal aprovada pelos avaliadores.

\subsection{Análise Estatística}

Para descrever a relação existente entre as características das amostras no teste de aceitação por escala hedônica utilizou-se a análise de variância (ANOVA) e teste de comparação de médias de Tukey, ao nível de significância de 5\%, histograma de frequência e Índice de Aceitabilidade (IA), segundo Dutcosky (2013).

Os resultados obtidos pelo CATA, foram avaliados conforme proposto por Santos e Cunha (2013) e Torres et al. (2017), utilizando o Teste $\mathrm{Q}$ de Cochran, para verificar diferença significativa $(\mathrm{p} \leq 0,05)$ entre os descritores sensoriais, eventualmente avaliar as diferenças entre as amostras, em seguida, foi realizada a Análise de Correspondência por meio de gráfico com as distâncias qui-quadrado da frequência de cada amostra entre os 12 termos descritores sensoriais.

Os dados de textura (TPA) foram avaliados por análise de variância (ANOVA), e teste de comparação de médias de Tukey ao nível de significância de 5\%, também foi realizada a Análise de Correspondência, para observar a correlação dos 12 descritores e das propriedades de textura no gráfico.

Utilizou-se para as análises o programa Statistica versão 10.0 (Statsoft, 2011).

\section{Resultados e Discussão}

A avaliação sensorial foi realizada em laboratório com 121 avaliadores não treinados, sendo 53\% do sexo feminino e 47\% masculino, com idades compreendidas entre 18 e 45 anos. As quatro amostras analisadas obtiveram aceitação com médias dos escores entre a 6,45 e 7,74 para todos os atributos avaliados, que correspondem a gostei moderadamente e gostei muito (Tabela 1). 
Tabela 1 - Médias dos escores de aceitação das amostras de requeijão tradicional e light.

\begin{tabular}{lcc|cc}
\hline \multirow{2}{*}{ Atributos } & \multicolumn{3}{c}{ Produto } \\
\cline { 2 - 5 } & TA & LA & TB & $6,96 \pm 1,64 \mathrm{~b}$ \\
\hline Impressão Global & $7,52 \pm 1,52 \mathrm{a}$ & $7,02 \pm 1,71 \mathrm{ab}$ & $7,17 \pm 1,43 \mathrm{ab}$ & $7,03 \pm 1,46 \mathrm{~b}$ \\
Aparência & $7,74 \pm 1,42 \mathrm{a}$ & $7,35 \pm 1,53 \mathrm{ab}$ & $7,18 \pm 1,35 \mathrm{~b}$ & $7,17 \pm 1,50 \mathrm{a}$ \\
Odor & $7,43 \pm 1,46 \mathrm{a}$ & $7,16 \pm 1,67 \mathrm{a}$ & $7,45 \pm 1,23 \mathrm{a}$ & $7,30 \pm 1,58 \mathrm{ab}$ \\
Textura & $7,48 \pm 1,60 \mathrm{~b}$ & $7,01 \pm 1,24 \mathrm{ab}$ & $7,59 \pm 1,24 \mathrm{a}$ & $6,55 \pm 2,11 \mathrm{bc}$ \\
Sabor & $7,25 \pm 1,91 \mathrm{a}$ & $6,45 \pm 2,06 \mathrm{c}$ & $7,18 \pm 1,74 \mathrm{ab}$ & \\
\hline
\end{tabular}

TA - Tradicional Marca A; LA - Light Marca A; TB - Tradicional Marca B; LB - Light Marca B. Médias seguidas da mesma letra na linha não diferem significativamente entre si $(\mathrm{p}<0,05)$. Fonte: Autores $(2020)$.

As diferenças $(\mathrm{p}<0,05)$ entre as amostras de requeijão foram observadas para os atributos: impressão global entre TA e LB; aparência de TA das amostras LA e LB; textura entre TA e TB; e o sabor entre TA e LA, e entre LA e TB. O único atributo que não apresentou diferença $(\mathrm{p} \geq 0,05)$ foi o odor.

Passos et al. (2017) avaliando requeijão cremoso de diferentes marcas comerciais obtiveram médias semelhantes às do presente estudo, identificando ainda que o gênero do consumidor influencia os resultados, pois ambos apresentam comportamentos distintos sendo que o gênero feminino percebeu diferenças significativas entre requeijões tradicionais, mas não entre amostras light; enquanto os avaliadores masculinos apresentaram resultados opostos.

Quanto ao Índice de Aceitabilidade, obteve-se resultados positivos com todas as amostras apresentando mais de $70 \%$ em todos os atributos avaliados (Figura 1).

Figura 1 - Índice de aceitabilidade por atributos para as amostras de requeijão tradicional e light.

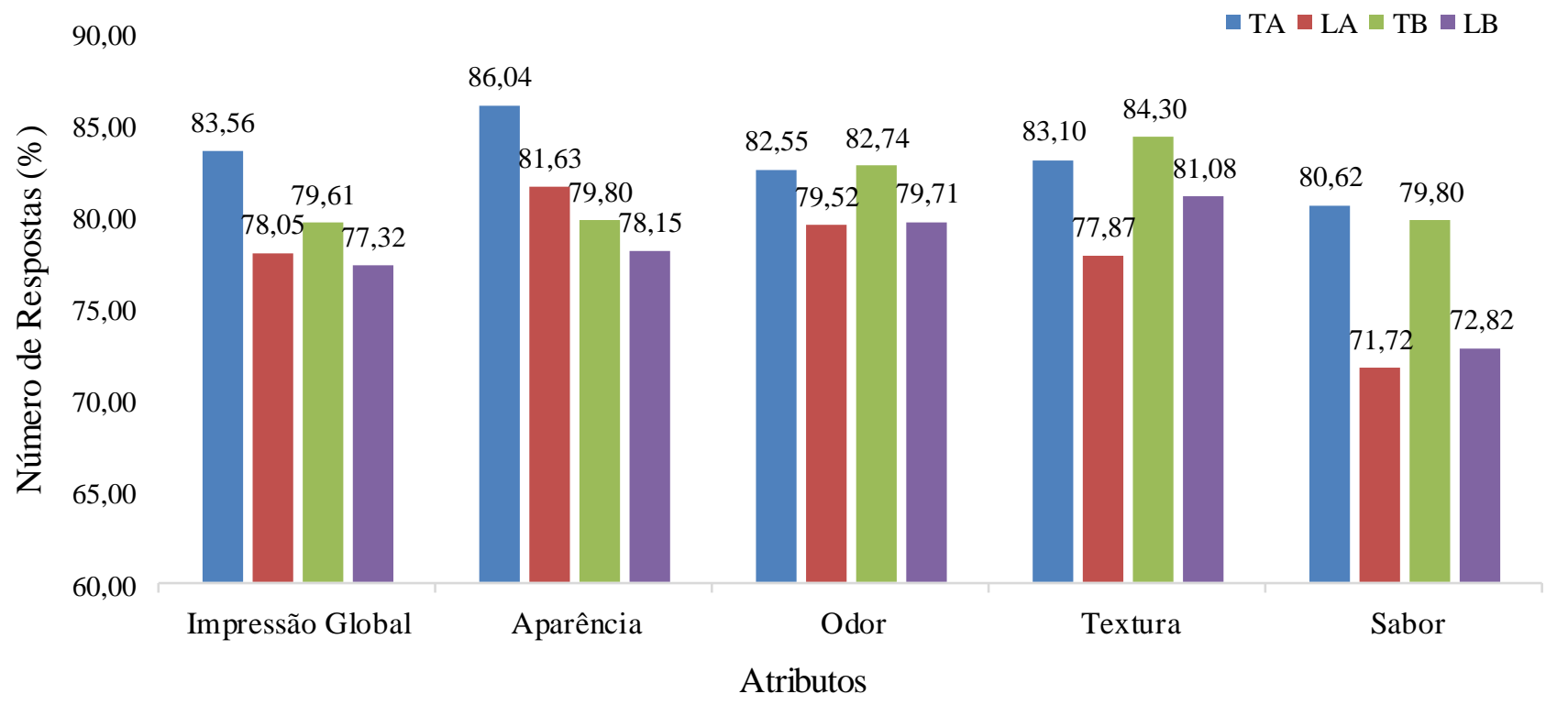

TA - Tradicional Marca A; LA - Light Marca A; TB - Tradicional Marca B; LB - Light Marca B. Fonte: Autores (2020).

Para a impressão global $(83,56 \%)$, aparência $(86,04 \%)$ e sabor $(80,62 \%)$, a amostra TA obteve maiores porcentuais, enquanto para os atributos odor $(82,74 \%)$ e textura $(84,30 \%)$ a amostra TB foi a mais aceita. Esses resultados indicam que as 
amostras de requeijão tradicionais ainda são mais aceitas pelos consumidores do que as amostras light, que possuem teor reduzido de gordura.

De acordo com os resultados do teste Q de Cochran, os avaliadores identificaram diferença significativa entre as amostras para cada termo sensorial abordado (Tabela 2).

Tabela 2 - Frequência das respostas dos descritores por atributos para as amostras de requeijão tradicional e light no teste CATA.

\begin{tabular}{|c|c|c|c|c|c|c|}
\hline \multirow{2}{*}{\multicolumn{2}{|c|}{$\begin{array}{c}\text { Termos } \\
\text { sensoriais }\end{array}$}} & \multicolumn{4}{|c|}{ Produto } & \multirow{3}{*}{$\begin{array}{c}\text { p-valor } \\
0,001\end{array}$} \\
\hline & & \multirow{2}{*}{$\begin{array}{c}\mathbf{T A} \\
108\end{array}$} & \multirow{2}{*}{$\begin{array}{l}\mathbf{L A} \\
112\end{array}$} & \multirow{2}{*}{$\begin{array}{c}\text { TB } \\
5\end{array}$} & \multirow{2}{*}{$\begin{array}{c}\mathbf{L B} \\
8\end{array}$} & \\
\hline & Cor Branca $^{0}$ & & & & & \\
\hline 可 & Cor Amarela ${ }^{0}$ & 7 & 7 & 102 & 101 & 0,000 \\
\hline$\frac{\pi}{4}$ & Massa Homogênea ${ }^{0}$ & 19 & 12 & 26 & 24 & 0,019 \\
\hline \multirow{3}{*}{$\ddot{0}$} & Ácido/Azedo ${ }^{0}$ & 8 & 35 & 10 & 13 & 0,000 \\
\hline & Característico Requeijão ${ }^{1}$ & 96 & 62 & 85 & 89 & 0,143 \\
\hline & Aroma Queijo $^{1}$ & 18 & 25 & 27 & 19 & 0,325 \\
\hline \multirow{3}{*}{$\underset{\mathscr{E}}{\stackrel{7}{e}}$} & Gorduroso $^{0}$ & 10 & 21 & 22 & 31 & 0,000 \\
\hline & Cremoso $^{1}$ & 64 & 92 & 86 & 82 & 0,366 \\
\hline & Firme $^{0}$ & 60 & 12 & 19 & 13 & 0,000 \\
\hline \multirow{3}{*}{$\stackrel{\check{\Xi}}{\mathscr{\mathscr { H }}}$} & Salgado $^{1}$ & 89 & 68 & 51 & 38 & 0,539 \\
\hline & Ácido ${ }^{0}$ & 25 & 52 & 10 & 8 & 0,000 \\
\hline & Adocicado $^{0}$ & 15 & 6 & 63 & 77 & 0,000 \\
\hline
\end{tabular}

TA - Tradicional Marca A; LA - Light Marca A; TB - Tradicional Marca B; LB - Light Marca B.

${ }^{0}$ Indica diferença significativa $(\mathrm{p}<0,05)$ de acordo com o teste $\mathrm{Q}$ de Cochran;

${ }^{1}$ Indica que não existe diferença significativa $(\mathrm{p} \geq 0,05)$.

Fonte: Autores (2020).

O teste estatístico examina se as variâncias das frequências apresentam homogeneidade entre as amostras frente aos descritores sensoriais, se são compatíveis e passíveis de comparação (Santos \& Cunha, 2013). Identificaram-se diferença $(\mathrm{p}<0,05)$ sensorial em 8 termos $(66,67 \%)$ dos 12 avaliados, reforçando a efetividade do avaliador no teste CATA. Caso similar foi observado por Torres et al. (2017) que obtiveram 18 termos, ao avaliar o desempenho do teste CATA e Teste Q de Cochran para novas abordagens estatísticas, usando três formulações de requeijões cremosos, que obtiveram 90,00\%, em um total de 20 termos.

No atributo Aparência, das quatro amostras (tradicional e light), 90,91\% consideraram a marca A no termo "Cor Branca" e 83,88\% a marca B para o termo "Cor Amarela". Houve uma variação $(12,81-20,60 \%)$ na frequência das respostas considerando as marcas no termo "Massa Homogênea", ou seja, nem branca e nem amarela. Contudo, os processos de fabricação do requeijão por marca são semelhantes.

No atributo Odor foi citado apenas o termo "Ácido/Azedo" entre as amostras LA e LB, que receberam as maiores frequências, 28,93\% (LA) e 10,74\% (LB), seguido das amostras tradicionais, com 8,26\% (TA) e 6,61\% (TB). Esse descritor revelou uma afinidade entre os processos nas diferentes marcas. 
Para o atributo Textura a marca A obteve $21,90 \%$ e a marca B $12,81 \%$ de frequência das respostas para o termo "Gorduroso", observando-se um comportamento semelhante ao do atributo Aparência onde a marca A se destacou em relação a marca B. Quanto ao descritor "Firme" as amostras light receberam os menores valores de frequência 9,92\% (LA) e 10,74\% (LB), já as amostras tradicionais os maiores valores 49,59\% (TA) e 15,70\% (TB).

No atributo Sabor, os dois termos citados "Ácido" e "Adocicado" se mostraram inversamente proporcionais, porém seguiram o mesmo comportamento apresentado nos atributos Aparência e Textura. Desse modo, entre as amostras tradicionais e light a maior frequência foi 31,82\% para marca A e a menor 7,44\% para marca B, em relação ao termo "Ácido". Logo, para o termo "Adocicado", a marca B recebeu a maior frequência de respostas, com 57,85\%, e a menor para marca A, com 8,68\%.

As diferenças existentes nas amostras comerciais de requeijão se devem às aplicações tecnológicas de produção, contudo, se observam semelhanças entre os produtos em alguns atributos. Os autores Garruti, Brito, Brandão, Uchôa Jr e Silva (2003) e Torres et al. (2017), perceberam que as características ou termos dos atributos Aparência, Textura e Sabor, são rigorosamente avaliados quando se trata da análise sensorial, isso quando avaliaram, também, requeijões comerciais entre tradicionais e light de marcas distintas.

Para avaliar a relação das amostras com os atributos do teste CATA, foi aplicada a Análise de Correspondência (Figura 2). É possível observar que a análise foi capaz de explicar $98,82 \%$ da variação, sendo o primeiro componente principal responsável por $82,59 \%$ e o segundo por $16,23 \%$.

Figura 2 - Análise de Correspondência das respostas do teste CATA para as amostras de requeijão tradicional e light.

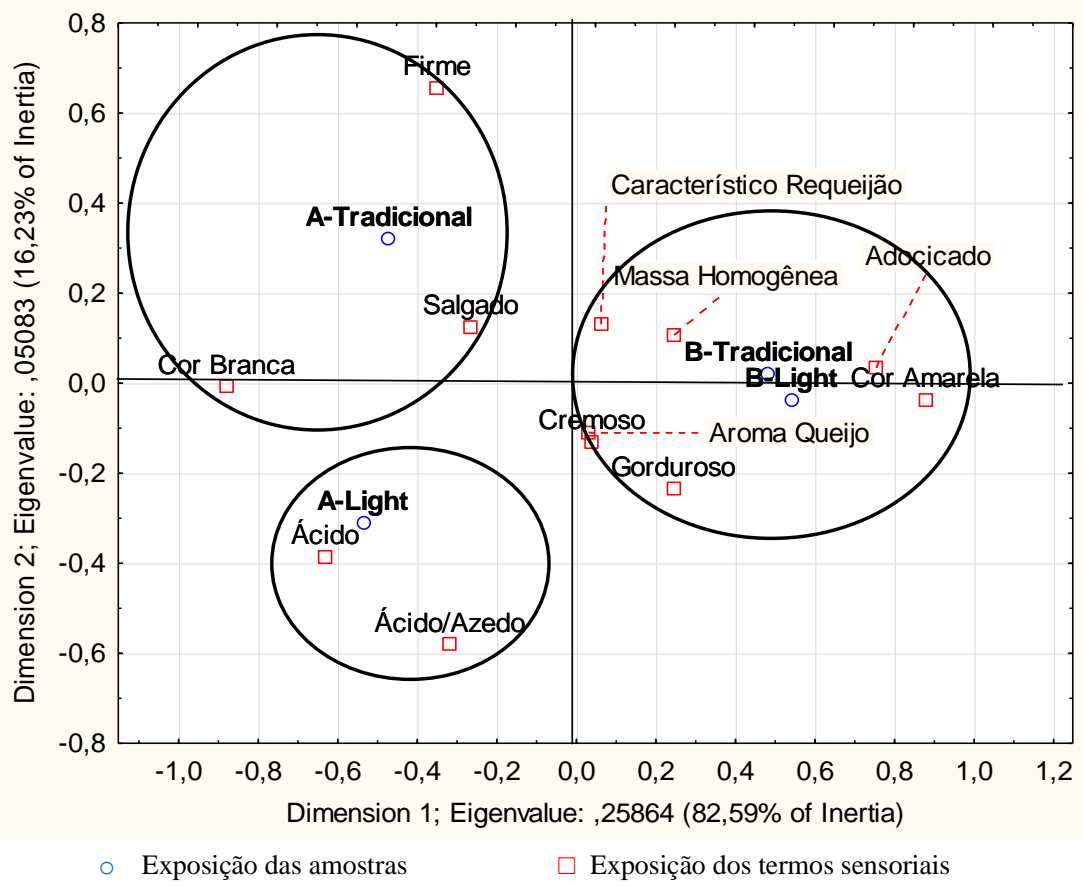

Fonte: Autores (2020).

A exposição espacial das quatro amostras propõe a formação de três grupos distintos: um primeiro grupo composto pelas amostras TB e LB sendo ambos da mesma marca, um segundo pela amostra TA e o terceiro pela amostra LA. As amostras da marca B (tradicional e light) estão alocadas no quadrante positivo, e se caracterizam por apresentar maior relação com os termos de "Massa Homogênea" e "Cor Amarela" (atributo aparência), "Característico de Requeijão" e "Aroma de Queijo" (atributo odor), "Gorduroso" e "Cremoso" (atributo textura), e "Adocicado" (atributo sabor). O segundo grupo formado pela amostra TA encontra-se no quadrante positivo, está correlacionado aos termos "Cor Branca" (atributo aparência), 
"Firme" (atributo textura), e "Salgado" (atributo sabor). Já a amostra LA está posicionada no quadrante negativo e apresenta uma maior relação com os descritores “Ácido/Azedo" (atributo odor) e "Ácido" (atributo sabor) (Figura 2).

No estudo de Torres et al. (2017), ao investigarem o desempenho da aplicação do CATA para obter o perfil sensorial de diferentes produtos de requeijão cremoso (tradicional; com amido; amido e óleo vegetal), a análise de componentes principais explicou aproximadamente $76 \%$ da variação, sendo o primeiro componente responsável por cerca $50 \%$, e segundo por aproximadamente $26 \%$. Desse modo, observa-se que o mapa percentual bidimensional é adequado para avaliar as relações entre as amostras/atributos, uma vez que explica grande parte da variabilidade dos dados, com mais de 50\% no primeiro componente tanto para essa pesquisa quanto para o estudo em questão.

Os descritores usados para caracterizar as amostras foram semelhantes aos relatados por Garruti et al. (2003) e Torres et al. (2017), que pesquisaram amostras comerciais de requeijão cremoso, descobrindo que os termos "Característico de Requeijão", “Adocicado" e "Cor Amarela" são especificidades importantes do requeijão tradicional cremoso. Ressalta-se que a distinção entre os atributos das amostras TA e TB pode ser decorrente da diferença entre as marcas comerciais, pois a tecnologia empregada no processamento do requeijão cremoso, segundo Minim et al. (2010), gera mudanças na viscosidade e consistência que são oriundas dos fatores físico-químicos, como temperatura, tamanho de partículas, pH, teor de sólidos e umidade.

Embora no primeiro grupo, a amostra LB se correlaciona com os atributos da amostra TB, observa-se que as amostras light não apresentaram o descritor "Firme". Minim, Simiqueli e Minim (2013) analisaram a influência do teor de gordura e umidade nas características sensoriais e na aceitação de requeijão light, observaram que atributos relacionados a textura (consistência, viscosidade e adesividade), apresentaram efeito negativo com relação ao teor de gordura, pois maiores níveis de gordura tornam o requeijão mais consistente, e como são light apresentam uma diminuição, o que acaba afetando a textura.

Para a análise instrumental de textura por TPA observa-se que o único parâmetro que não demonstrou diferença significativa $(\mathrm{p} \geq 0,05)$ entre as amostras foi a elasticidade (Tabela 3$)$.

Tabela 3 - Médias dos parâmetros texturais das amostras de requeijão tradicional e light por TPA.

\begin{tabular}{|c|c|c|c|c|}
\hline \multirow{2}{*}{ Propriedades de Textura } & \multicolumn{4}{|c|}{ Produtos } \\
\hline & TA & LA & TB & LB \\
\hline Firmeza (N) & $273,64 \pm 4,14 a$ & $144,97 \pm 1,66 \mathrm{~d}$ & $247,26 \pm 4,80 b$ & $220,04 \pm 4,18 \mathrm{c}$ \\
\hline Adesividade (J) & $-387,40 \pm 11,37 d$ & $-91,63 \pm 1,73 a$ & $-282,30 \pm 2,71 \mathrm{c}$ & $-143,34 \pm 2,40 b$ \\
\hline Elasticidade (mm) & $0,980 \pm 0,01 \mathrm{a}$ & $0,989 \pm 0,01 \mathrm{a}$ & $0,983 \pm 0,00 \mathrm{a}$ & $0,985 \pm 0,01 \mathrm{a}$ \\
\hline Coesividade & $0,962 \pm 0,02 \mathrm{a}$ & $0,932 \pm 0,01 \mathrm{a}$ & $0,924 \pm 0,02 b$ & $0,944 \pm 0,02 \mathrm{a}$ \\
\hline Gomosidade (N) & $273,48 \pm 1,44 a$ & $173,39 \pm 1,85 \mathrm{~d}$ & $199,25 \pm 1,79 \mathrm{c}$ & $209,14 \pm 3,75 b$ \\
\hline Mastigabilidade (J) & $271,66 \pm 8,52 \mathrm{a}$ & $134,44 \pm 0,78 \mathrm{~d}$ & $196,40 \pm 1,83 c$ & $213,05 \pm 4,61 b$ \\
\hline
\end{tabular}

TA - Tradicional Marca A; LA - Light Marca A; TB - Tradicional Marca B; LB - Light Marca B. Médias seguidas da mesma letra na linha não diferem significativamente entre si $(\mathrm{p}<0,05)$. Fonte: Autores (2020).

O estudo de Treszczynska, Siwek, Kowalik, Lobacz e Zulewska (2018), investigou as preferências dos consumidores em relação aos queijos tvarog, obtendo resultados semelhantes para a análise de TPA, pois não houve diferença significativa na elasticidade para todas as amostras analisadas, ressalta-se que essa elasticidade é calculada a partir da diferença entre a altura da amostra antes e após o primeiro ciclo de compressão. Firmeza, adesividade, gomosidade e mastigabilidade indicaram diferença significativa $(\mathrm{p}<0,05)$, quanto a coesividade, a amostra TB diferiu das demais analisadas. 
Esses resultados indicam que a diferença no processamento existente entre o requeijão light ( $25 \%$ menos gordura que o requeijão tradicional), influencia diretamente nas características texturais. Salienta-se ainda, que os parâmetros do TPA variam conforme o tamanho e forma da amostra, razão entre a sonda e a amostra, grau de compressão, taxa de deformação, número de repetições e mastigação (Fogaça, Silva \& Rodrigues, 2017).

A Análise de Correspondência também foi usada para avaliar a relação das amostras com as respostas da textura no teste sensorial CATA e a caracterização de TPA (Figura 3). A análise foi capaz de explicar 95,87\% da variação, sendo o primeiro componente principal responsável por $86,49 \%$ e o segundo por $9,38 \%$.

Figura 3 - Análise de Correspondência do teste CATA e da caracterização de TPA das amostras requeijão tradicional e light.

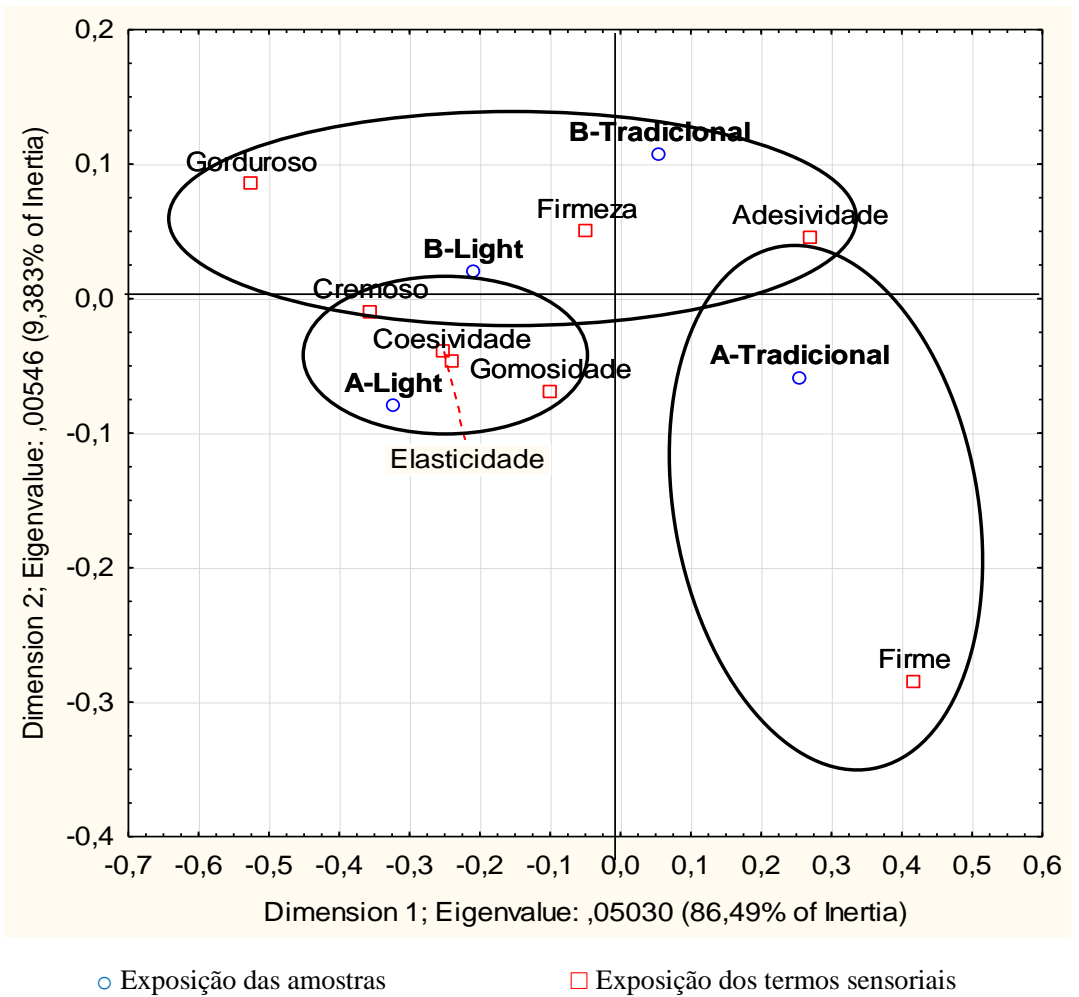

Fonte: Autores (2020).

No que diz respeito a exposição espacial das quatro amostras, sugere-se a formação de três grupos diferentes: um primeiro grupo contemplado pelas amostras TB e LB, um segundo pela amostra LA e o terceiro pela amostra TA. As amostras TB e LB estão dispostas no quadrante positivo, e identificam-se por apresentar maior relação com os termos: "Firmeza" e "Adesividade" (para TPA), e "Gorduroso" e "Cremoso" (para textura - CATA). O segundo grupo formado pela amostra LA encontra-se no quadrante negativo, estando correlacionado aos termos "Coesividade", "Gomosidade" e "Elasticidade" para TPA, e "Cremoso" para textura no teste CATA. E o terceiro grupo que corresponde a amostra TA está posicionada no quadrante negativo e apresenta uma única relação.

Segundo Torres et al. (2017), a caracterização sensorial de requeijão cremoso pelo consumidor empregando o CATA quando correlacionada com a Análise Descritiva que utiliza múltiplos fatores, foi capaz de explicar 75\% da variação, sendo o primeiro componente responsável por aproximadamente $52 \%$ e o segundo por $23 \%$, evidenciando assim, que ambos os métodos são capazes de fornecer informações equivalentes no que diz respeito aos atributos sensoriais das amostras. Assim, é possível inferir que o CATA pode ser usado como método sensorial apropriado, seguro e de baixo custo para o estabelecimento do perfil sensorial de formulações de requeijão. 
A análise sensorial é usada como uma ferramenta científica para mensurar e compreender as constatações humanas a estímulos externos, envolvendo aspectos psicológicos e fisiológicos, e quando associada aos procedimentos instrumentais, proporciona uma forma mais rápida da percepção humana. Porém, a análise instrumental vem sendo usada em pesquisas na textura dos alimentos, pois requer menor custo, economiza tempo, apresenta resultados numéricos e oportunidade de correlacionar com a análise sensorial (Fogaça et al., 2017).

Assim, ao correlacionar as respostas do teste CATA e a caracterização de TPA, percebe-se de forma geral, que as amostras apresentaram características de textura de requeijão cremoso, que segundo Van Dender (1992), as principais são: firmeza, elasticidade, coesividade, gomosidade e mastigabilidade. Observa-se ainda, que a amostra LA encontra-se em quadrante oposto as demais, assim, pode-se inferir que o procedimento tecnológico usado para elaboração da formulação light alterou de forma perceptível seus atributos de textura segundo a TPA em relação as outras amostras, e que os avaliadores foram capazes de discriminar a amostra LA adequadamente das amostras TA e TB pelo teste CATA, portanto a relação entre as respostas do teste sensorial CATA e caracterização de TPA foram adequadamente correlacionadas.

A Análise de Correspondência entre o teste sensorial CATA e a caracterização de TPA, indicou quais os descritores estão correlacionados com as amostras para ambas as análises. Assim, a indústria de alimentos pode optar por usar o teste sensorial em conjunto com os dados instrumentais, para avaliar a aprovação de atributos em uma determinada amostra, reduzindo assim, tempo de fabricação e o custo (Souza, Pereira, Gomes \& Carneiro, 2011).

Todavia, em uma visão geral, o processamento do requeijão tradicional e light é semelhante em todas as características sensoriais dos produtos que se encontram no mercado. Em seus estudos, Silva et al. (2012) e Masson, Viganó e Bordignon (2016) obtiveram resultados similares, ao desenvolverem e analisarem sensorialmente requeijão cremoso tradicional, light com teor de sódio reduzido, e com substituto de gordura, quando comparados com requeijões comerciais.

\section{Conclusão}

A aceitabilidade das marcas de requeijão comercial tradicional e light demonstrou não haver diferença apenas em relação ao atributo odor.

A correlação das medidas instrumentais e sensoriais no quesito textura indicou que a marca B, independente do processo, apresenta as mesmas características (descritores), já na marca A, foi perceptível a influência do teor de gordura no processamento, pois os participantes distinguiram o requeijão LA do TA, evidenciando que o processo de fabricação permitiu a diferenciação quanto à textura e os atributos sensoriais do requeijão.

Como a aceitabilidade é influenciada pelo valor do produto, se recomenda para estudos futuros a avaliação de custo considerando a elaboração tradicional e light.

\section{Agradecimentos}

Ao Instituto Federal de Educação, Ciência e Tecnologia do Ceará (IFCE) Campus Limoeiro do Norte pelo suporte de laboratórios e materiais de análise e a Coordenação de Aperfeiçoamento de Pessoal de Nível Superior (CAPES) pelo apoio financeiro e suporte de bolsas para o Mestrado em Tecnologia em Alimentos do IFCE.

\section{Referências}

Alcantara, M. de, \& Freitas-Sá, D. de G. C. (2018). Metodologias sensoriais descritivas mais rápidas e versáteis - uma atualidade na ciência sensorial. Brazilian Journal of Food Technology, 21(e2016179), 1-12. https://doi.org/10.1590/1981-6723.17916

Amaral, S. M. B., da Silva, F. S., da Silva Freitas, R. V., de Almeida Souza, M. J., Mendes, L. G., \& Cavalcante, A. B. D. (2020). Microbiological and physical chemical evaluation of traditional cream cheese brands with different market value. Research, Society and Development, 9(10), e1439108262e1439108262. https://doi.org/10.33448/rsd-v9i10.8262 
Brasil (1997). Ministério da Agricultura e do Abastecimento. Departamento de Inspeção de Produtos de Origem Animal. Portaria $n^{\circ} 359$ de 04 de setembro de 1997. Regulamento Técnico para Fixação de Identidade e Qualidade do Requeijão Cremoso ou Requesón. Diário Oficial [da] República Federativa do Brasil. Brasília, DF. Seção 1, p. 19690.

Brasil (2013). Ministério da Saúde. Resolução $n^{o} 466$ do Conselho Nacional de Saúde, de 12 de dezembro de 2012. Aprova as diretrizes e normas regulamentadoras de pesquisas envolvendo seres humanos. Diário Oficial [da] União, Brasília, DF. Seção 1, p. 59.

Bourne, M. C. (1978). Texture profile analysis. Food Technology, 32(7), 62-66.

Cervo, A. L., Bervian, P. A. \& Da Silva, R. (2007). Metodologia científica. (6a ed.), Pearson Prentice Hall, 162 p.

Dutcosky, S. D. (2013). Análise sensorial de alimentos. (4a ed.), Champagnat, 531 p.

Embrapa. (2020a). Especial Coronavírus - Pesquisa de Consumo de Lácteos. https://www.cileite.com.br/especial_coronavirus_pesquisa_consumo. Acesso em: 27 fev. 2021

Embrapa. (2020b). Anuário leite 2020: leite de vacas felizes. https://www.embrapa.br/busca-de-publicacoes/-/publicacao/1124722/anuario-leite-2020-leite-devacas-felizes.

Fogaça, D. N. L., da Silva, W. S., \& Rodrigues, L. B. (2017). Influence of compression parameters on mechanical behavior of mozzarella cheese. Journal of texture studies, 48(5), 427-432. https://doi.org/10.1111/jtxs.12246

Garruti, D. D. S., Brito, E. S. D., Brandão, T. M., Uchôa Jr, P., \& da Silva, M. A. A. (2003). Desenvolvimento do perfil sensorial e aceitação de requeijão cremoso. Food Science and Technology, 23(3), 434-440. https://doi.org/10.1590/s0101-20612003000300024

Lima, K. R., Medeiros, J. S., Leão, P. V. T., da Cunha, J. V. T., de Freitas Borges, W., de Oliveira Santos, G., ... \& Nicolau, E. S. (2021). Perfil físico-químico e sensorial de requeijão cremoso obtido a partir de diferentes coagulantes. Research, Society and Development, 10(2), e33710212455-e33710212455. https://doi.org/10.33448/rsd-v10i2.12455

Masson, A. P., Viganó, O. J., \& Bordignon, S. (2016). Requeijão cremoso de copo com teor reduzido de sódio e enriquecido com fibras. Revista E-Tech: Tecnologias para Competitividade Industrial, 9(1), 123-149. https://doi.org/10.18624/e-tech.v9i1.483

Minim, V. P. R., dos Santos Navarro, R. D. C., Milagres, M. P., Martins, E. M. F., Sampaio, S. C. S. A., \& Vasconcelos, C. M. (2010). Contribuição dos atributos sensoriais para aceitabilidade de requeijão cremoso. Revista do Instituto de Laticínios Cândido Tostes, 65(372), 34-42.

Minim, V. P. R., Simiqueli, A. A., \& Minim, L. A. (2013). Influence of fat and moisture content in the processing of light requeijão. Journal of Food Research, 2(6), 12-23. https://doi.org/10.5539/jfr.v2n6p12

Passos, F. R., Araújo, W. D. B., Ribeiro, L., Teodoro, R. A. R., Fernandes, R. V. B., \& Mendes, F. Q. (2017). Avaliação sensorial de requeijão cremoso de diferentes marcas comerciais. The Journal of Engineering and Exact Sciences, 3(6), 769-773. https://doi.org/10.18540/jcecvl3iss6pp0769-0773

Pereira, A. S., Shitsuka, D. M., Parreira, F. J., \& Shitsuka, R. (2018). Metodologia da pesquisa científica. UFSM. https://repositorio.ufsm.br/bitstream/handle/1/15824/Lic_Computacao_MetodologiaPesquisaCientifica.pdf?sequence=1.

Santos, E., \& Cunha, M. (2013). Interpretação crítica dos resultados estatísticos de uma meta-análise: Estratégias metodológicas. Millenium-Journal of Education, Technologies, and Health, (44), 85-98.

Silva, R. de C. dos S. N. da, Minim, V. P. R., Lima, L. P. de, Gomide, A. I., Moraes, L. E. da S., \& Minim, L. A. (2012). Otimização da aceitabilidade sensorial de requeijão cremoso light. Ciência Rural, 42(2), 360-366. https://doi.org/10.1590/S0103-84782012000200027

Souto, V. O., Santos, E. B. S., Alves, N. S., Nascimento, V. F., \& Silva, A. R. (2017). Análise instrumental em salsicha: cor e textura. Revista Brasileira de Agrotecnologia, 7(2), 280-283.

Souza, V. R. de, Pereira, P. A. P., Gomes, U. J., \& Carneiro, J. de D. S. (2011). Avaliação e definição do perfil de textura ideal de queijo Petit Suisse. Revista do Instituto de Laticínios Cândido Tostes, 66(382), 48-53.

Statsoft (2011). Statistica for window: computer programa manual. Versão 10.0, Tulsa: Statsoft Inc.

Torres, F. R., Esmerino, E. A., Carr, B. T., Ferrão, L. L., Granato, D., Pimentel, T. C., \& Cruz, A. G. (2017). Rapid consumer-based sensory characterization of requeijão cremoso, a spreadable processed cheese: Performance of new statistical approaches to evaluate check-all-that-apply data. Journal of Dairy Science, 100(8), 6100-6110. https://doi.org/10.3168/jds.2016-12516

Treszczynska, B., Siwek, A., Kowalik, J., Lobacz, A., \& Zulewska, J. (2018). Influence of flavors and stabilizers used on the preferences and properties of aerated tvarog curd cheese. Acta Scientiarum Polonorum Technologia Alimentaria, 17(4), 335-346. https://doi.org/10.17306/j.afs.2018.0591

Trintim, L. T., Castilho, L. G., Rosa, N. C., Marco, R. de; Oliveira, R. F. F. de, Paraíso, C. M., Santos, S. S. dos; \& Madrona, G. S. (2017). Desenvolvimento e aceitação sensorial de requeijão cremoso com adição de óleo essencial de orégano. Brazilian Journal of Surgery and Clinical Research, 21(1), 38-42.

Van Dender, A. G. F. (1992). Princípios básicos da fabricação de queijos fundidos e requeijão cremoso. Revista Leite e Derivados, 1(3), 26. 